\title{
Synthesis of Multiwall Carbon Nanotube/Graphene Composite by an Aerosol Process and its Characterization for Supercapacitors
}

\author{
Eun Hee Jo", ${ }^{1,2}$, Sun Kyung Kim ${ }^{1}$, Hankwon Chang ${ }^{1,2}$, Chongmin Lee ${ }^{1}$, Su-Ryeon Park ${ }^{1}$, \\ Ji-Hyuk Choi ${ }^{2}$, Hee Dong Jang ${ }^{1,2^{*}}$ \\ ${ }^{1}$ Resources Utilization Research Center, Korea Institute of Geoscience and Mineral Resources, Yuseong-gu, Daejeon \\ 34132, Korea \\ ${ }^{2}$ Department of Nanomaterials Science and Engineering, University of Science and Technology, Yuseong-gu, Daejeon \\ 34113, Korea
}

\begin{abstract}
A multiwall carbon nanotube/graphene (MWCNT/GR) composite was synthesized for an enhanced supercapacitor. Aerosol spray drying (ASD) was used to synthesize the MWCNT/GR particles using a mixture of graphene oxide (GO) solution and MWCNT. The effect of the MWCNT/GO ratio on the properties of the composite, including its shape and structure, was investigated. The composite particles were generally shaped like a crumpled paper ball, with an average diameter of approximately $5 \mu \mathrm{m}$. The MWCNTs, which were uniformly dispersed among the graphene sheets, not only increased the basal spacing of the sheets but also bridged the wide gaps between them, thereby improving electron transfer between the layers. Thus, the MWCNTs increased the contact area of the electrolyte/electrode and facilitated the transportation of electrons and electrolyte in the electrode. Using a two-electrode testing system, the electrochemical results demonstrate that the MWCNT/GR (weight ratio $=0.1$ ) electrode has a capacitance of $192 \mathrm{~F} \mathrm{~g}^{-1}$ and an excellent rate of capacity retention $\left(88 \%\right.$ at $\left.4 \mathrm{~A} \mathrm{~g}^{-1}\right)$.
\end{abstract}

Keywords: Supercapacitor; MWCNT/GR; Aerosol spray drying.

\section{INTRODUCTION}

As the demand for energy storage systems with higher energy and power density has increased, supercapacitors have attracted much attention as a next-generation ecofriendly energy storage device. These supercapacitors have higher power density and higher charge/discharge efficiency than conventional secondary batteries, as well as semipermanent cycle life and a low risk of explosion because they are stable to current changes. Therefore, supercapacitors are largely used as power supplies for instantaneous acceleration with motors and memory backup of personal electronic devices, supplementary batteries, and electric

\footnotetext{
* Corresponding author.

Tel.: +82-42-868-3612; Fax: +82-42-868-3415

E-mail address: hdjang@kigam.re.kr
}

This article is an English version of "Synthesis of Multiwall Carbon Nanotube/Graphene Composite by Aerosol Process and its Characterization for Supercapacitors" published in Particle and Aerosol Research in December 2016. vehicles that require stable energy supply (Frackowiak and Beguin, 2001; Miller and Simon, 2008).

The electrochemical performance of a supercapacitor can be determined by the electrode materials, which requires high conductivity, large specific surface area, high temperature stability, uniform pore structure, and low cost. Currently, carbon-based materials, such as carbon nanotube (Frackowiak and Beguin, 2002), activated carbon (Zhu et al., 2011), graphene (Zhang et al., 2010; Xu et al., 2011), etc., are widely employed as electrode materials in supercapacitors. Among electrode materials, activated carbon is the most frequently used material due to its large surface area and low-cost. However, limited energy storage capacity and rate capacity restrict its applications (Kim et al., 2012). Therefore, graphene based materials, including 0D fullerenes (zero-dimensional fullerenes), 1D CNTs (one-dimensional carbon nanotubes), and 2D/3D GR (two-dimensional/threedimensional graphene), are of particular interest because of their unique structures and exceptional electrical and mechanical properties (Allen et al., 2010; Shao et al., 2015). In addition to supercapacitors, such graphene are also attractive materials for use in the fields of electrochemical sensors and solar cells (Jo et al., 2013; Kim et al., 2014).

Recently, structural approaches to increasing the performance of graphene capacitors have been reported 
(Luo et al., 2013; Zhang et al., 2013). However, these electrodes have lower volume capacitance than conventional activated carbon electrodes due to the severe stacking of graphene in the electrode. As an approach for improving the volume capacitance of the supercapacitor, many works have been carried out on the production of graphene composites including carbon materials such as CNTs and activated carbon (Lu et al., 2011; Huang et al., 2015). However, as re-stacking and agglomeration occur between the graphene sheets of the two-dimensional structure, the electrolyte is not able to effectively penetrate the composite electrode. In addition, the assembly of carbon nanotubes by Van der Waals attraction limited the excellent electrical conductivity and unique pore structure, which tended to decrease the specific capacitance value with increasing current. To overcome these problems, it is necessary to enhance the capacitance value and power density with high current density.

Here, we introduce a 3D multiwall carbon nanotube/graphene (MWCNT/GR) composite as the electrode for high-performance supercapacitors. Using aerosol spray drying, the MWCNT/GR composite was prepared from graphene oxide (GO) and MWCNT mixtures. During preparation, MWCNT served to reduce the degree of aggregation of the GO layers during configuration of the 3D structure of MWCNT/GR composites. In particular, MWCNTs loaded on the edges and on the surface of the graphene layers are expected to accelerate the electrolyte accessibility due to enhancement of the electrical conductivity and to an increase in the layer spacing by cross-linking the graphene sheets. We analyzed the properties (morphology, interlayer spacing, defects) of MWCNT/GR composites using SEM, TEM, and Raman analysis according to the mixing ratio of GO and MWCNT. Then, we examined the electrochemical properties such as the specific capacitance and the rate capability of the as-fabricated MWCNT/GR electrodes for supercapacitors.

\section{METHODS}

\section{Synthesis of MWCNT/GR Composites}

The graphene oxide (GO) was synthesized by the oxidation of graphite using the modified Hummers' method (Hummers and Offeman, 1958; Cote et al., 2009). The functionalization of MWCNTs was conducted using an earlier reported method (Byon et al., 2011) to increase the dispersion of MWCNT (>95\% purity; NanoLab, Inc.) in deionized water. $1 \mathrm{~g}$ of MWCNTs was dispersed in a mixed solution of $150 \mathrm{~mL}$ of $\mathrm{H}_{2} \mathrm{SO}_{4}(99.5 \%)$ and $50 \mathrm{~mL}$ of $\mathrm{HNO}_{3}$, followed by stirring at $70^{\circ} \mathrm{C}$ for $2 \mathrm{~h}$. Thereafter, it was filtered and washed with $5 \% \mathrm{HCl}$ solution, and then dried in air. Acid-treated MWCNTs and GO were mixed at a weight ratio of 0.01 to 0.5 to prepare starting solutions. The concentration of GO in the colloidal mixture was fixed at $0.25 \mathrm{wt} \%$. The MWCNT/GO composites were prepared using an aerosol reactor equipped with air flow nozzles, and the schematic illustration of the reaction is displayed in Fig. 1. This aerosol reactor is composed of a two-fluid nozzle for the injection of the starting solution, a drying chamber, a bag filter, and a cyclone collector. The starting solution was sprayed into a drying chamber kept at the temperature of $200^{\circ} \mathrm{C}$. The as-dried sample was collected using a series of a cyclone and a bag filter. As-prepared MWCNT/GO composites were then further heated at $250^{\circ} \mathrm{C}$ for $2 \mathrm{~h}$ in an argon atmosphere.

\section{Preparation of the Supercapacitor Assembly}

To examine the electrochemical performance of the supercapacitors, we first made an electrode using polyvinylidene difluoride (PVDF) as binder and MWCNT/GR composites as active electrode materials. The weight fractions of the composite and PVDF were maintained at 90 and $10 \%$, respectively. A mixture of composite and PVDF was stirred for $20 \mathrm{~min}$ while adding $N$-methyl-2-pyrrolidone (NMP) to obtain uniform slurry mix. The mixture was then pasted onto carbon paper $10 \mu \mathrm{m}$ thick using the doctor blade method and then dried under vacuum at $80^{\circ} \mathrm{C}$ for $2 \mathrm{~h}$. After drying, the paper was cut to form a circle with a diameter of $14 \mathrm{~mm}$ for a cell that weighed $5 \mathrm{mg}$. The supercapacitor cell was assembled using a two-electrode HS Flat Cell (Hohsen Corp., Japan) with a separator (Whatman Grade GF/C Glass Microfiber) between it and the electrolyte $(5 \mathrm{M} \mathrm{KOH})$.

\section{Analysis}

The surface morphology of MWCNT/GR composites with different MWCNT loadings was observed using a field emission scanning electron microscope (FESEM; Sirion, FEI) and transmission electron microscopy (TEM; JEM-ARM200F, JEOL). The crystal structure and interlayer spacing of the composites were analyzed by X-ray diffractometry (XRD; SmartLab, Rigaku Co.). The defects

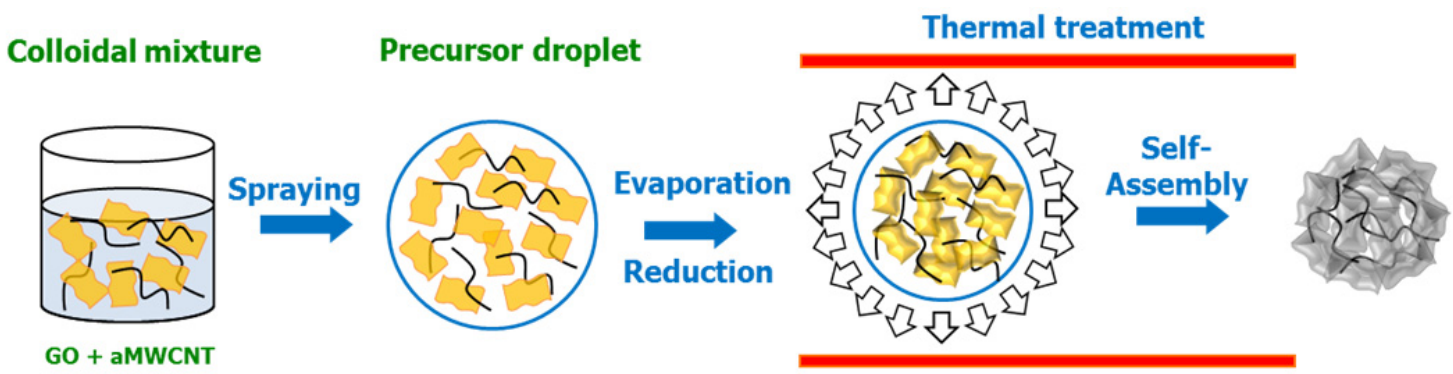

Fig. 1. Schematic illustration of the formation of MWCNT/GR composite from a colloidal mixture of MWCNT and GO via aerosol spray drying. 
of graphene were investigated by Raman spectra (Lambda Ray, Dimension P1, Lambda Solutions, Inc., Korea). The electrochemical behavior of the MWCNT/GR electrodes was characterized by constant current charge/discharge and impedance measurements with two electrode cells in an HS Flat Cell (Hohsen Corp., Japan) using an electrochemical interface instrument (VSP, Bio-Logics, USA).

\section{RESULTS AND DISCUSSION}

Fig. 2 exhibits FESEM and TEM images of the assynthesized MWCNT/GR composites with various MWCNT loading. The images confirmed that the MWCNT/GR composites had a 3D morphology, which appeared as crumpled paper balls that were about $5 \mu \mathrm{m}$ in diameter. The shape did not change with increasing MWCNT content in the composite particles. The existence of MWCNT in the sample was confirmed using a TEM image, and the MWCNT contents on the surface of graphene sheets increased with increasing weight ratio of MWCNT/GO. The MWCNT/GR composites with a weight ratio of $0.01-$ 0.1 showed that the MWCNT was uniformly distributed
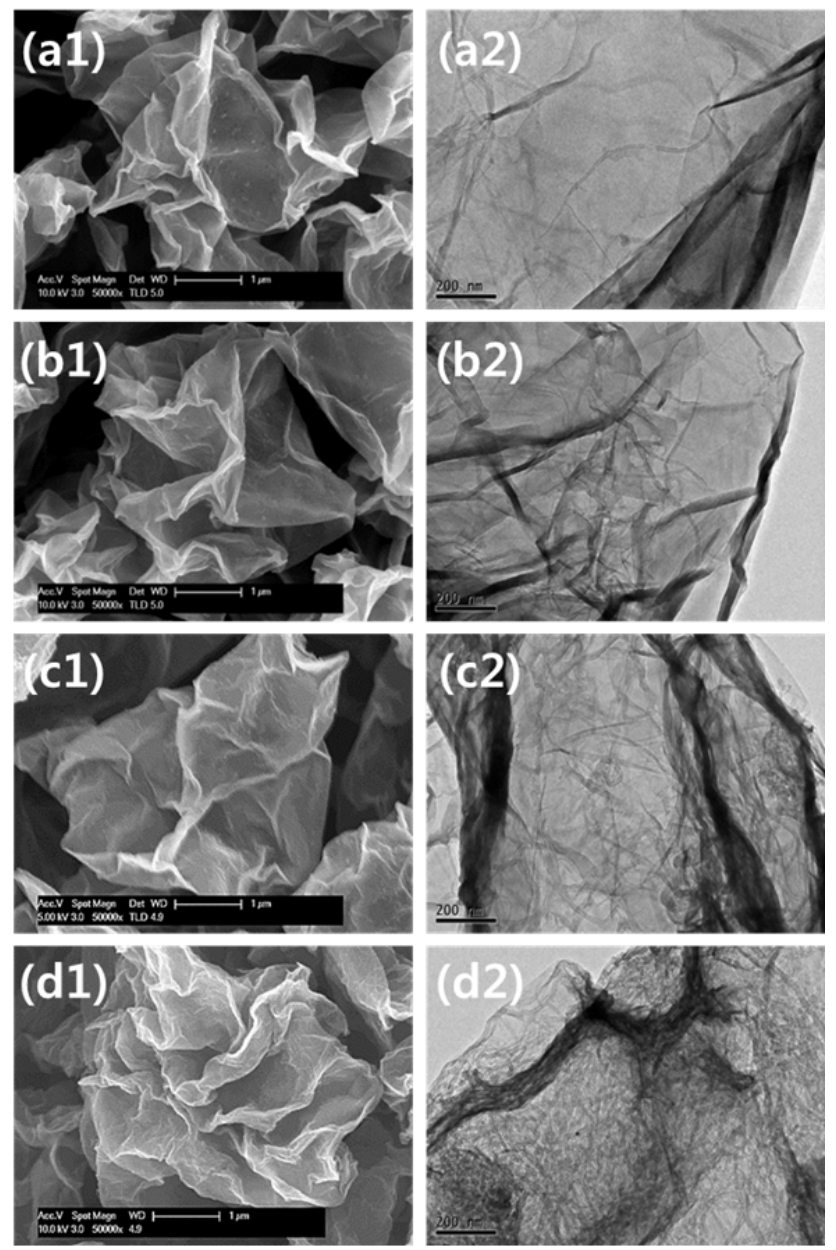

Fig. 2. FESEM and TEM images of the MWCNT/GR composites prepared at different $\mathrm{MWCNT} / \mathrm{GO}$ ratios: (a1, a2) 0.01; (b1, b2) 0.05; (c1, c2) 0.1; (d1, d2) 0.5 (@) GO: $0.25 \mathrm{wt} \%$ ). between the graphene layers. However, in the case of the MWCNT/GR composite having a weight ratio of 0.5 , it was confirmed that the MWCNTs aggregated and formed a bundle when the MWCNT content was high in the graphene sheet.

The XRD results of the MWCNT/GR are exhibited in Fig. 3. The XRD peaks of the MWCNT/GR composites exhibit two diffraction peaks at $2 \theta=23.5^{\circ}$ and $42.9^{\circ}$ or $2 \theta$ $=26.4^{\circ}$ and $42.9^{\circ}$, indicating the co-existence of graphene sheets and MWCNTs. The peak of GO at $10^{\circ}$ was not observed in the XRD patterns of composites, suggesting that the reduction of GO was performed well during preparation of the composite. The peaks of MWCNT/GR composites in the XRD patterns became broad and shifted left compared to the GO peak, which indicates loosely stacked graphene and increase in the interfacial spacing by the interaction of MWCNTs in the composite particles. The interlayer spacing (calculated from Bragg's law) of MWCNT/GR composites with weight ratios of $0.01,0.05$, 0.1 , and 0.5 was $0.37,0.37,0.36$ and $0.34 \mathrm{~nm}$, respectively (see Eq. (1)) (Lu et al., 2011):

$$
d_{002}=\frac{n \lambda}{2 \sin \theta}
$$

where $d$ is the interplanar distance, $\theta$ is the degree of diffraction, and $\lambda$ is the wavelength of incident ray, $n$ is an integer.

These results indicate that the introduction of MWCNT causes an increase of the interlayer spacing of the graphene sheet. On the other hand, in the case of a sample having a weight ratio of 0.5 , it can be seen that the interlayer spacing was reduced compared to other MWCNT/GR composites. This suggests that increase in the amount of MWCNT induced aggregation between MWCNTs, thus reducing the interlayer spacing of the composites.

Fig. 4 presents the Raman scattering spectrum of the MWCNT/GR samples. For the as-synthesized composites, D-band and G-band of graphene were confirmed at 1350 and $1600 \mathrm{~cm}^{-1}$, respectively. The D-band is related to the disorder that arises from $\mathrm{sp}^{3}$ atom or other contaminant, while the G-band expresses the $\mathrm{sp}^{2}$ carbon atom of graphite. The ratio of intensity of D-band and G-band $\left(\mathrm{I}_{\mathrm{D}} / \mathrm{I}_{\mathrm{G}}\right)$ was generally considered to represent the amount of disordered carbon relative to graphitic carbon. The $\mathrm{I}_{\mathrm{D}} / \mathrm{I}_{\mathrm{G}}$ ratio of the composites decreased as the weight ratio of MWCNT/GO increased. It is considered that defects in the composite were reduced as the MWCNT content increased.

To acquire further insight into the effect of various contents of MWCNTs on the electrochemical performance, we prepared supercapacitors in a symmetrical two-electrode system. Fig. 5(a) provides the CV (cyclic voltammetry) of different samples, obtained at a scan rate of $10 \mathrm{mV} \mathrm{s}^{-1}$. The CV curves of MWCNT/GR electrodes were all close to an ideal rectangular shape, with no obvious redox current peak, over the range $0-1.0 \mathrm{~V}$, indicating standard EDLC (electrical double layer capacitor) behavior. The rectangular shape expanded with increase of the MWCNT/GR weight 


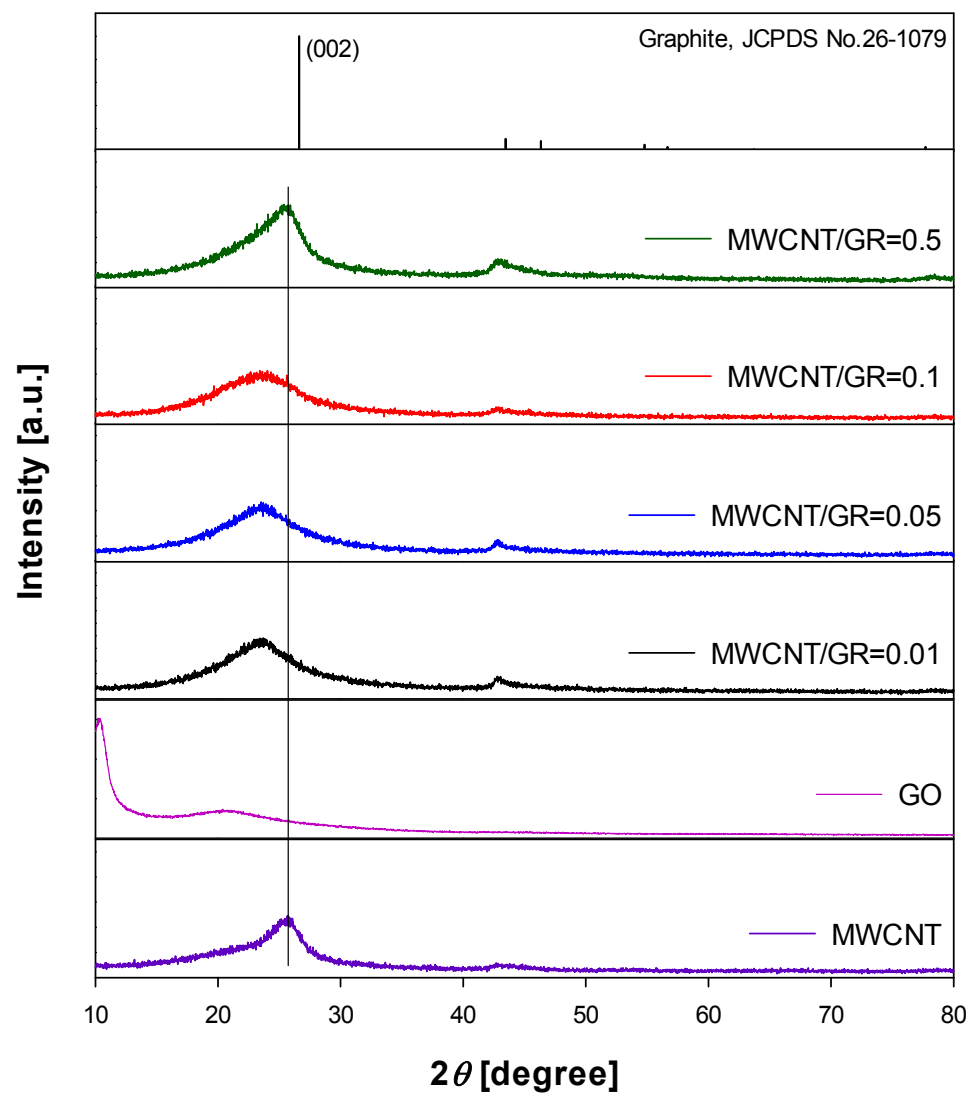

Fig. 3. X-ray diffraction patterns of the MWCNT, GO, and MWCNT/GR composites prepared at different MWCNT/GO ratios: 0.01,0.05,0.1, and 0.5 (@ GO: 0.25 wt\%).

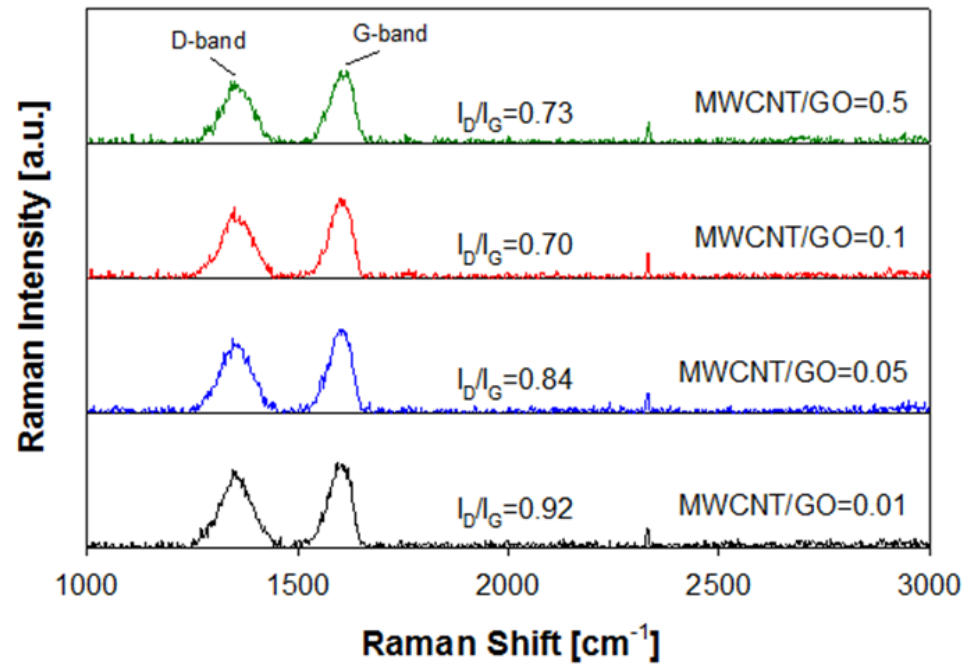

Fig. 4. Raman spectra of the MWCNT/GR composites prepared at different MWCNT/GO ratios: $0.01,0.05,0.1$, and 0.5 (@) GO: $0.25 \mathrm{wt} \%$ ).

ratio from 0.01 to 0.1 , which indicated that the electrode's specific capacitance was enhanced. It was thought that the introduction of MWCNTs induced an increase of interlayer spacing between graphenes, which better enabled the electrolyte ions to penetrate to the surface of the electrode material. On the other hand, when the weight ratio of $\mathrm{MWCNT} / \mathrm{GO}$ is 0.5 , it is considered that the aggregation between the MWCNTs rapidly reduced the interlayer spacing of the graphene. This seems to have affected the specific capacitance as well as slowing the ion transfer rate of the electrolyte.

On the basis of the galvanostatic charge/discharge curves line, the specific capacitance of the MWCNT/GR electrodes on different current density were calculated and 

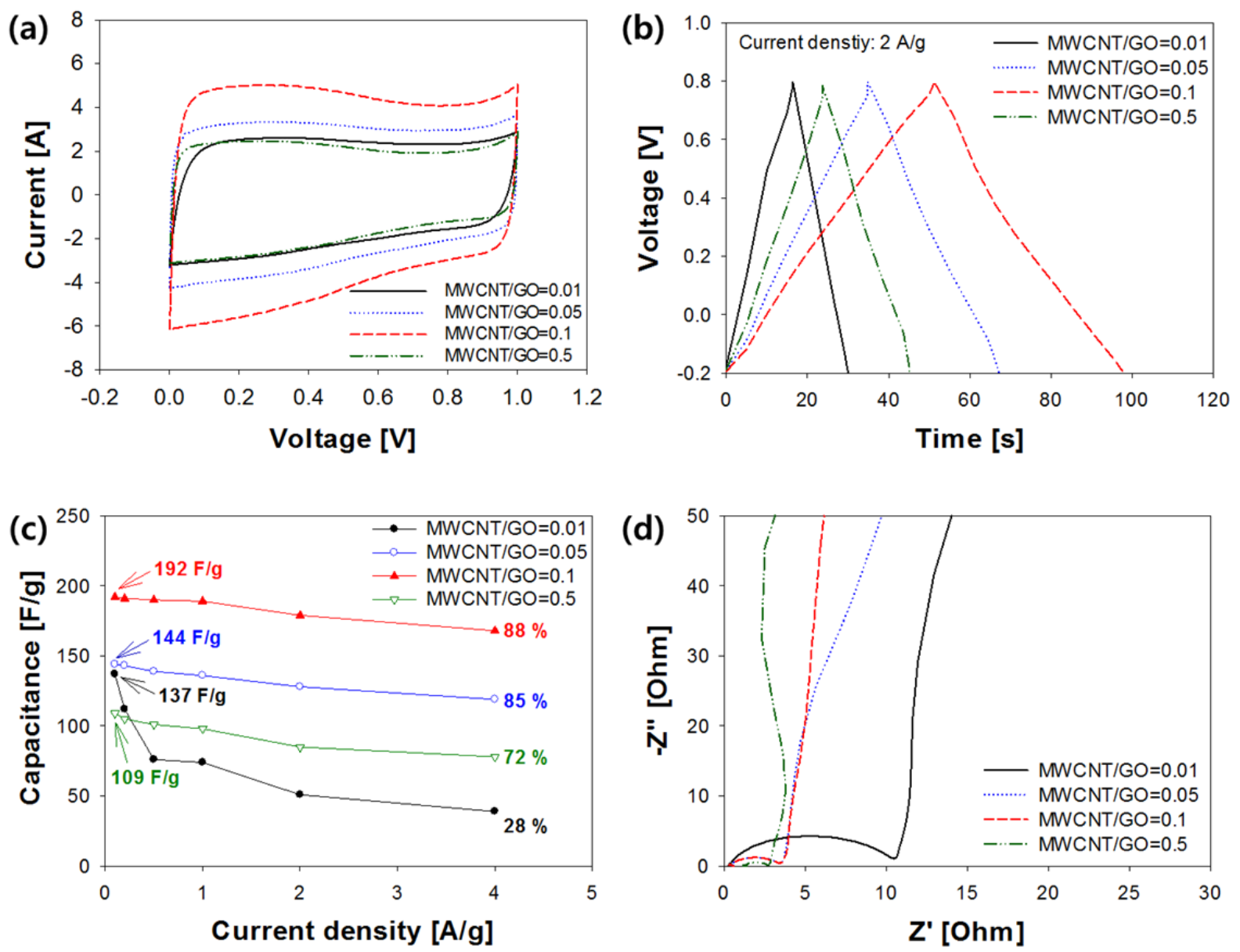

Fig. 5. Electrochemical properties of electrodes prepared with different MWCNT/GR composites: (a) CV curves recorded at a scan rate of $10 \mathrm{mV} \mathrm{s}^{-1}$; (b) Galvanostatic charge/discharge curves at a current density of $2 \mathrm{~A} \mathrm{~g}^{-1}$; (c) Specific capacitance variation at different current density; (d) Nyquist plots of these electrodes.

expressed in Figs. 5(b)-5(c). As a result of charge/discharge tests, all electrodes showed symmetrical charge/discharge curves indicating reversibility, and the specific capacity can be obtained using Eq. (2):

$C_{p}=\frac{4 I \Delta t}{m \Delta V}$

where I (A) is the discharge current for the applied time duration $\Delta \mathrm{t}(\mathrm{s}), \mathrm{m}(\mathrm{g})$ is the mass of the composite in one electrode, and $\Delta \mathrm{V}(\mathrm{V})$ is the potential range. The specific capacitance calculated from the discharge process at a current of $0.1 \mathrm{~A} \mathrm{~g} \mathrm{~g}^{-1}$ for the MWCNT/GR samples were $137,144,192$, and $109 \mathrm{~F} \mathrm{~g}^{-1}$, respectively. As the weight ratio increased from 0.1 to 0.5 , the specific capacitance also increased, but the specific capacitance of the MWCNT/GR electrode having a weight ratio of 0.5 decreased sharply. In the case of the MWCNT/GR composite with a weight ratio of 0.1 , it was confirmed that the specific capacitance remained high as the scan rate increased. From these results, we found that there are optimum conditions for the weight ratio of MWCNT and GO. As mentioned in the previous $\mathrm{CV}$ results, the introduction of MWCNT facilitated the penetration of electrolyte ions by increasing the interlayer spacing between the graphene sheets. Therefore, we confirmed that the specific capacitance of MWCNT/GR electrodes was maintained despite the increase of the scan rates. However, the specific capacity of the electrode fabricated at a weight ratio of 0.5 was low at all current densities. As can be shown from the TEM images, introduction of excessive MWCNT seems to increase the bundles between the CNTs in the graphene sheets and reduce the specific capacitance due to increase in the interfacial resistance.

On the other hand, an impedance test was conducted to investigate the interface resistance into the electrode/ electrolyte. As the weight ratio of MWCNT/GO increases, the bulk resistance (the intercept value of the $\mathrm{Z}$-axis in Fig. 5(d)) is similar except at the MWCNT/GR ratio of 0.5 . However, the interfacial resistance (the semicircle's diameter in the Nyquist plot) decreased with increasing MWCNT content. This is because the fast electrical conductivity of the MWCNTs decreased the resistance of the electrode. In addition, the increase in the interlayer spacing between the graphene layers highly increased the contact area of the electrolyte, thereby greatly reducing the interfacial resistance. 


\section{CONCLUSIONS}

In this study, an MWCNT/GR composite fabricated by drying aerosol spray was used to improve the stability of the power density of supercapacitors. The as-prepared composite was a powder composed of 3D spherical particles with an average size of $5 \mu \mathrm{m}$ and was uniformly dispersed among the graphene layers. Investigating the performance of the EDLC made from the MWCNT/GR composite revealed that the highest specific capacity, $192 \mathrm{~F} \mathrm{~g}^{-1}$, was achieved with an MWCNT/GO weight ratio of 0.1 . Therefore, we consider that the introduction of MWCNTs into graphene sheets can improve properties such as the electrical conductivity, penetration of electrolyte ions, and interlayer spacing of the sheets. In particular, the ability to maintain a high specific capacitance at high current densities is due to the fact that MWCNTs efficiently form cross-linking points within the graphene sheets. In addition, it is thought that the pores in the three-dimensional MWCNT/GR composite reduced the interfacial resistance between the electrode and the electrolyte.

\section{ACKNOWLEDGMENTS}

This research was supported by the Basic Research Project of the Korea Institute of Geoscience and Mineral Resources (KIGAM), funded by the Ministry of Science and ICT.

\section{DISCLAIMER}

The authors declare no conflicts of interest.

\section{REFERENCES}

Allen, M.J., Tung, V.C. and Kaner, R.B. (2010). Honeycomb carbon: A review of graphene. Chem. Rev. 110: $132-145$.

Byon, H.R., Lee, S.W., Chen, S., Hammond, P.T. and Shao-Horn, Y. (2011). Thin films of carbon nanotubes and chemically reduced graphenes for electrochemical micro-capacitors. Carbon 49: 457-467.

Cote, L.J., Cruz-Silva, R. and Huang, J.X. (2009). Flash reduction and patterning of graphite oxide and its polymer composite. J. Am. Chem. Soc. 131: 11027-11032.

Frackowiak, E. and Beguin, F. (2001). Carbon materials for the electrochemical storage of energy in capacitors. Carbon 39: 937-950.

Frackowiak, E. and Beguin, F. (2002). Electrochemical storage of energy in carbon nanotubes and nanostructured carbons. Carbon 40: 1775-1787.

Huang, N.B., Kirk, D.W., Thorpe, S.J., Liang, C.H., Xu, L.S., Li, W., Zhang, S.C. and Sun, M. (2015). Effect of Carbon nanotube loadings on supercapacitor characteristics. Int. J. Energy Res. 39: 336-343.

Hummers, W.S. and Offeman, R.E. (1958). Preparation of graphitic oxide. J. Am. Chem. Soc. 80: 1339-1339.

Jo, E.H., Kim, S.K., Jang, H.D., Chang, H., Roh, K.M. and Kim, T.O. (2013). Preparation of grapheme-TiO composite by aerosol process and it's characterization for dye-sensitized solar cell. Part. Aerosol Res. 9: 5157.

Kim, J., Kwon, Y., Lee, J.K. and Choi, H.S. (2012). Influence of oxygen-/nitrogen-containing functional groups on the performance of electrical double-layer capacitor. Korean Chem. Eng. Res. 50: 1048-1043.

Kim, S.K., Jang, H.D., Chang, H. and Choi, J.W. (2014). Preparation of graphene-palladium composite by aerosol process and it's characterization for glucose biosensor. Part. Aerosol Res. 10: 53-59.

Lu, X.J., Dou, H., Gao, B., Yuan, C.Z., Yang, S.D., Hao, L., Shen, L.F. and Zhang, X.G. (2011). A flexible graphene/multiwalled carbon nanotube film as a high performance electrode material for supercapacitors. Electrochim. Acta 56: 5115-5121.

Luo, J.Y., Jang, H.D. and Huang, J.X. (2013). Effect of sheet morphology on the scalability of graphene-based ultracapacitors. ACS Nano 7: 1464-1471.

Miller, J.R. and Simon, P. (2008). Materials science Electrochemical capacitors for energy management. Science 321: 651-652.

Shao, Y.L., El-Kady, M.F., Wang, L.J., Zhang, Q.H., Li, Y.G., Wang, H.Z., Mousavi, M.F. and Kaner, R.B. (2015). Graphene-based materials for flexible supercapacitors. Chem. Soc. Rev. 44: 3639-3665.

Xu, B., Yue, S.F., Sui, Z.Y., Zhang, X.T., Hou, S.S., Cao, G.P. and Yang, Y.S. (2011). What is the choice for supercapacitors: Graphene or graphene oxide? Energy Environ. Sci. 4: 2826-2830.

Zhang, L., Zhang, F., Yang, X., Long, G., Wu, Y., Zhang, T., Leng, K., Huang, Y., Ma, Y., Yu, A. and Chen, Y. (2013). Porous 3D graphene-based bulk materials with exceptional high surface area and excellent conductivity for supercapacitors. Sci. Rep. 3: 1408.

Zhang, L.L., Zhou, R. and Zhao, X.S. (2010). Graphenebased materials as supercapacitor electrodes. J. Mater. Chem. 20: 5983-5992.

Zhu, Y., Murali, S., Stoller, M.D., Ganesh, K.J., Cai, W., Ferreira, P.J., Pirkle, A., Wallace, R.M., Cychosz, K.A., Thommes, M., Su, D., Stach, E.A. and Ruoff, R.S. (2011). Carbon-Based supercapacitors produced by activation of graphene. Science 332: 1537-1541.

Received for review, October 17, 2018 Revised, October 17, 2018 Accepted, November 19, 2018 